\title{
Validity of Electron Temperature Measurement by Using Boltzmann Plot Method in Radio Frequency Inductive Discharge in the Atmospheric Pressure Range
}

\author{
Noriyasu OHNO, M. Abdur RAZZAK ${ }^{1)}$, Hiroshi UKAI ${ }^{1)}$, \\ Shuichi TAKAMURA ${ }^{1)}$ and Yoshihiko UESUGI ${ }^{2)}$ \\ EcoTopia Science Institute, Nagoya University, Nagoya 464-8603, Japan \\ ${ }^{1)}$ Department of Energy Engineering and Science, Graduate School of Engineering, \\ Nagoya University, Nagoya 464-8603, Japan \\ ${ }^{2)}$ Department of Electrical and Electronic Engineering, Graduate School of Natural Science, \\ Kanazawa University, Kanazawa 920-1192, Japan
}

(Received 25 November 2005 / Accepted 5 April 2006)

\begin{abstract}
The validity of electron temperature measurement using the Boltzmann plot method was investigated in a radio frequency (rf) inductive discharge in the atmospheric pressure range. Since the Boltzmann plot method requires local thermal equilibrium (LTE), the parameter regions of electron temperature and electron density, in which the Boltzmann plot method is applicable, were analyzed in terms of a comparison of the spontaneous emission and collisional excitation to the excited states. It is observed that in our rf-inductive plasma near the atmospheric pressure range the Boltzmann plot method does not provide an exact measurement of electron temperature because of relatively low electron density, which does not satisfy the calculation criteria. An alternative method employing a combination of the Stark broadening measurement and the Saha-Boltzmann equation was demonstrated to more accurately determine the plasma parameters (electron temperature and electron density).
\end{abstract}

(C) 2006 The Japan Society of Plasma Science and Nuclear Fusion Research

Keywords: rf thermal plasma, spectroscopy, thermodynamic equilibrium, Boltzmann plot, atmospheric pressure discharge

DOI: $10.1585 /$ pfr. 1.028

\section{Introduction}

Thermal plasmas are currently considered one of the most effective and attractive tools for breaking down waste materials and disposing of harmful gases $\left(\mathrm{CO}_{2}, \mathrm{SF}_{6}\right.$, etc. $)$. However, although thermal plasmas are used for the disposal of low-level radioactive waste and the decomposition of chlorofluorocarbon (CFC), halogen, PCBs, etc. in Japan and in many developed countries throughout the world, such plasmas' processing cost, which is high compared with conventional techniques, has led to its limited use. However, in contrast to conventional processing techniques, the disposal/decomposition process using thermal plasmas does not produce harmful auxiliary substances such as $\mathrm{NO}_{\mathrm{x}}, \mathrm{SO}_{\mathrm{x}}$, dioxin, etc. Due to this advantage, thermal plasmas have become very attractive in comparison to recent and past conventional disposal techniques [1-7]. In addition, thermal plasmas have proven to be a very attractive tool for both the thermal treatment of powders and material synthesis due to the high-temperature (typically $>$ $10,000 \mathrm{~K}$ ), high-reaction activity, and lack of environmentally unfriendly side effects [8-11].

Recently, radio frequency rf-inductive plasmas in the atmospheric pressure range have been developed using a

author'se-mail: ohno@ees.nagoya-u.ac.jp static induction transistor (SIT) inverter power source [1217]. However, in order to use these rf-inductive plasmas in industrial and environmental applications, it is necessary to measure the plasma parameters (electron temperature, $T_{\mathrm{e}}$, and electron density, $n_{\mathrm{e}}$ ) as a way to elucidate the quality of the generated plasmas.

To evaluate the electron temperature and density of low-pressure plasmas, probe measurements are widely used [18-21]. However, a probe theory for atmospheric pressure plasmas having a large electron-neutral collision frequency has not yet been established. Furthermore, the application of probe measurement to atmospheric pressure plasmas are difficult due to the problems regarding large heat load and high-frequency noise. Therefore, as an alternative to probe measurement, optical methods using spectral line emission intensity are widely used to measure internal plasma parameters $\left(T_{\mathrm{e}}\right.$ and $\left.n_{\mathrm{e}}\right)$ in the atmospheric pressure range. Recently, the electron temperature of rfinductive Ar plasmas in the atmospheric pressure range has been measured based on the line intensity ratio of the visible spectrum by employing the Boltzmann plot method, assuming that the plasma is in a local thermodynamic equilibrium (LTE) condition [22-26]. However, the measurement criteria along with the LTE condition, which must be 
satisfied in order to apply the Boltzmann plot method for calculating the electron temperature, have not been verified in these studies. The present paper investigates the validity of using the Boltzmann plot method to measure electron temperature in a rf-inductive discharge in the atmospheric pressure range.

Here, the electron temperature of rf-inductive Ar plasmas, $T_{\mathrm{e}}$, is measured first using the spectral intensity of the ArI line at a wavelength range of $547-570 \mathrm{~nm}$ using the Boltzmann plot method, and the electron density, $n_{\mathrm{e}}$, is then also evaluated based on the Stark broadening measurement. Next, an alternative method using the combination of the Stark broadening measurement and the Saha-Boltzmann equation is demonstrated to determine the plasma parameters $\left(T_{\mathrm{e}}\right.$ and $\left.n_{\mathrm{e}}\right)$ near the atmospheric pressure range, and these values are compared with those determined using the Boltzmann plot method and the Stark broadening method, respectively.

\section{Experimental Setup}

In the present experiment, an Optical Multi-channel Analyzer (OMA) spectroscopic system is used for the optical measurement of the plasma parameters $\left(T_{\mathrm{e}}\right.$ and $\left.n_{\mathrm{e}}\right)$. The complete system consists of a Czerny-Turner type spectrometer (SPEX: $750 \mathrm{M}$ ), a two-dimensional CCD camera (Princeton Instruments: CCD-1100PB), and an optical fiber, as shown in Fig. 1. The specifications of the spectroscopic system are shown in Table 1. The optical emission radiated from the plasma is transmitted to the OMA using the optical fiber through a lens having a focal length of $110 \mathrm{~mm}$ and a diameter of $60 \mathrm{~mm}$. The lens is mounted $100 \mathrm{~mm}$ away from the center of the plasma. By using the CCD camera controller (Princeton Instruments: ST138), the data of the measured radiation intensities of the plasma spectrum were stored in a personal computer using the software WinSpec 1.3B.

Radio frequency inductive plasmas were generated in a device known as a RIP (RF Inverter Plasma torch) [12-17]. The plasmas were sustained in a cylindrical Pyrex glass chamber having an inner diameter of $70 \mathrm{~mm}$ and length of $200 \mathrm{~mm}$ by applying a rf power from a static induction transistor (SIT) inverter power source. The frequency and output power rating of the SIT inverter were $0.5-1.5 \mathrm{MHz}$ and $20 \mathrm{~kW}$ (pulse operation), respectively. An induction coil consisting of 7 turns of a copper tube having a $1 / 4$ inch outer diameter was used as the loop antenna. The rf power level, which is limited by the cooling capability of the system, was modulated with a $200 \mathrm{~ms}$ square-wave pulse without the use of a cooling system. An $L C$ matching network was employed to optimize the power coupling. The internal plasma parameters $\left(T_{\mathrm{e}}\right.$ and $\left.n_{\mathrm{e}}\right)$ of rfinductive Ar plasmas were measured at gas pressures of 70 , 80 , and $90 \mathrm{kPa}$ with a fundamental resonance frequency of $1.100 \mathrm{MHz}$ and at a gas flow rate of $18 \mathrm{lpm}$. The spectral intensities of the ArI lines radiated from Ar plasmas at

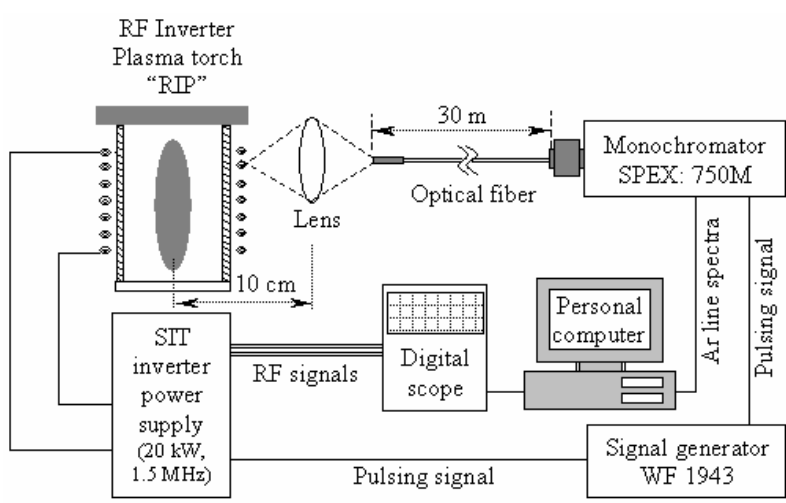

Fig. 1 Optical system for spectroscopic measurement. The system consists of an OMA, signal generator, digital oscilloscope, personal computer, and plasma production device ("RIP") along with a SIT inverter power source.

Table 1 Specifications of spectroscopic system.

\begin{tabular}{|l||l|r|}
\hline \multirow{4}{*}{$\begin{array}{l}\text { Spectro meter } \\
\text { (SPEX 750M) }\end{array}$} & Focal length & $0.75 \mathrm{~m}$ \\
\cline { 2 - 3 } & Measured range & $0-1500 \mathrm{~nm}$ \\
\cline { 2 - 3 } & Grating & 1200 Groove $/ \mathrm{mm}$ \\
\cline { 2 - 3 } & Dispersion & $1.1 \mathrm{~nm} / \mathrm{mm}$ \\
\cline { 2 - 3 } & Bridge wavelength & $400 \mathrm{~nm}$ \\
\hline CCD camera & Resolution & $1100 \times 330$ pixel \\
\hline \multirow{3}{*}{ Optical fiber } & Applicable range & Visible \\
\cline { 2 - 3 } & Material & Quartz \\
\cline { 2 - 3 } & Core diameter & $400 \mathrm{~nm}$ \\
\cline { 2 - 3 } & Length & $30 \mathrm{~m}$ \\
\hline
\end{tabular}

a wavelength range of 547-570 $\mathrm{nm}$ were measured during 150-200 ms in a $200 \mathrm{~ms}$ plasma. The slit width and height of the spectrometer were adjusted to $20 \mu \mathrm{m}$ and $50 \mathrm{~mm}$, respectively. In order to set the shutter timing of the CCD camera of the OMA, the triggering signal was applied to the CCD camera and to the rf source simultaneously.

\section{Electron Temperature Measure- ment by Boltzmann Plot Method}

\subsection{Basic theory}

The Boltzmann plot method is a simple and widely used method for spectroscopic measurement, especially for measuring the electron temperature of plasma from using the relative intensity of a two or more line spectra having a relatively large energy difference. However, in order to practically apply the Boltzmann plot method for the measurement of electron temperature, the excitation level needs to be reached under a LTE condition. The basic principle of the Boltzmann plot method is described below [27].

Let us consider two energy levels, $E_{i}$ and $E_{j}$ ( $E_{i}$ for lower level and $E_{j}$ for upper level), of two different line intensities with respective atomic densities of $N_{i}$ and $N_{j}$. Under the thermal equilibrium condition, the relation between $N_{i}$ and $N_{j}$ can be established using the Boltzmann 
distribution:

$$
\frac{N_{j}}{N_{i}}=\frac{g_{j}}{g_{i}} \exp \left[-\frac{E_{j}-E_{i}}{k T}\right],
$$

where $g_{i}, g_{j}$ are the statistical weights of the respective states, $k$ is the Boltzmann constant $\left(1.38 \times 10^{-23} \mathrm{~J} / \mathrm{K}\right)$, and $T$ is the temperature in $\mathrm{K}$. If the total population density is $N$, the Boltzmann relation applicable to the population distribution over a given atomic state is given by

$$
\frac{N_{j}}{N}=\frac{g_{j}}{Z(T)} \exp \left[-\frac{E_{j}-E_{i}}{k T}\right],
$$

where $Z(T)$ is the sum of the weighted Boltzmann function of all the discrete energy levels, which is called the partition function given by

$$
Z(T)=\sum_{m} g_{m} \exp \left[-\frac{E_{m}}{k T}\right] .
$$

Now, when the plasma atom is de-excited from the upper energy level, $E_{j}$, to the lower energy level, $E_{i}$, the emission coefficient of spectral line can be expressed by the following equation:

$$
\varepsilon_{j i}=\frac{h c_{0}}{4 \pi \lambda_{j i}} A_{j i} N_{j},
$$

where $\lambda_{j i}$ is the wavelength of the emitted light, $h$ is the Planck's constant $\left(6.626 \times 10^{-34} \mathrm{~J} \cdot \mathrm{s}\right), c_{0}$ is the velocity of light in vacuum $\left(3 \times 10^{8} \mathrm{~m} / \mathrm{s}\right)$, and $A_{j i}$ is the transition probability, which is the probability per second that an atom in state $j$ spontaneously emits in a random direction and is deexcited to state $i$. Putting Eq. (4) in Eq. (2) and rearranging them gives

$$
\frac{\varepsilon_{j i} \lambda_{j i}}{A_{j i} g_{j}}=\frac{h c_{0} N}{4 \pi Z(T)} \exp \left[-\frac{E_{j}}{k T}\right] .
$$

Taking the logarithm in both sides of Eq. (5) gives

$$
\ln \frac{\varepsilon_{j i} \lambda_{j i}}{A_{j i} g_{j}}=-\frac{E_{j}}{k T}+C,
$$

where $C$ is a constant.

Now, plotting Eq. (6) with $E_{j}$ in the horizontal axis and $\ln \left(\varepsilon_{j i} \lambda_{j i} /\left(A_{j i} g_{j}\right)\right)$ in the vertical axis will result in a straight line, and the electron temperature can be determined from the slope of the straight line.

\subsection{Applicable regime of the Boltzmann plot method for ArI line}

In order to apply the Boltzmann plot method to determine the electron temperature, the Boltzmann distribution needs to be thermalized at the target excitation level. Since it is assumed that collisional excitation is predominant in high-pressure radio frequency inductive plasmas, the Boltzmann plot method for electron temperature measurement is applicable when the following condition is fulfilled [28]:

$$
C_{i j} \gg A_{j},
$$

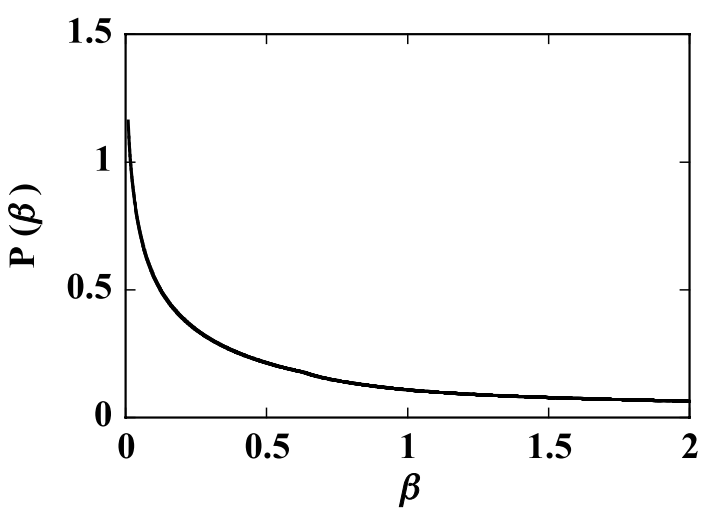

Fig. $2 P(\beta)$ as a function of $\beta$.

where $A_{j}$ represents the transition probability from level $j$ to the ground state, and $C_{i j}$ represents the collisional excitation coefficient, which is given by

$$
C_{i j}=n_{\mathrm{e}}\left\langle\sigma_{\mathrm{e}} v\right\rangle
$$

with $\left\langle\sigma_{\mathrm{e}} v\right\rangle$ being the rate coefficient given by [28]

$$
\left\langle\sigma_{\mathrm{e}} v\right\rangle=3.2 \times 10^{-7} f_{i j}\left(\frac{R_{\mathrm{y}}}{\Delta E}\right)^{3 / 2} \beta^{1 / 2} \mathrm{e}^{-\beta} P(\beta),
$$

where $\Delta E\left(=E_{j}-E_{i}\right)$ is the energy difference between level $j$ and $i$ in $\mathrm{eV}, R_{\mathrm{y}}$ is the Rydberg constant $(10.9 \times$ $\left.10^{7} \mathrm{~m}^{-1}\right), f_{i j}$ is the oscillator strength given by

$$
f_{i j}=\frac{g_{j}}{g_{i}} \frac{2 \pi \varepsilon_{0} m_{\mathrm{e}} c_{0}^{3} A_{j i}}{e^{2} \hbar^{2} \Delta E^{2}}=\frac{g_{j}}{g_{i}} \frac{A_{j i}}{4.310 \times 10^{7} \Delta E^{2}},
$$

and $P(\beta)$, which as a function of $\beta$ is plotted in Fig. 2, given by $\beta=\Delta E / T$.

The parameter region of electron temperature and electron density, where the Boltzmann plot method is applicable, has been analyzed by comparison between transition probability, $A_{j}$, and collisional excitation to the excited states, $C_{i j}$, using the condition mentioned in Eq. (7). Here the condition $C_{i j}>10 A_{j}$ is applied to investigate the domain of the Boltzmann plot method. Figure 3 shows the parameter region of the Boltzmann plot method for measuring the electron temperature and electron density of rf-inductive Ar plasmas from the spectral line intensities emitted from an argon atom (ArI $555.87 \mathrm{~nm}$ ) with a transition probabiity, $A_{j}$, of $2 \times 10^{8} \mathrm{~s}^{-1}$. Generally, in rfinductive plasmas in the atmospheric pressure range, the values of the electron temperature range are found to range from 7,000-10,000 K [22-26]. Figure 3 shows that the Boltzmann plot method can be applied in an electron density range of $10^{23}-10^{24} \mathrm{~m}^{-3}$ with an electron temperature range of 7,000-10,000 K.

\subsection{Electron temperature of rf-inductive Ar plasma}

The electron temperature of $\mathrm{rf}$-inductive Ar thermal plasmas has been evaluated using the Boltzmann plot 


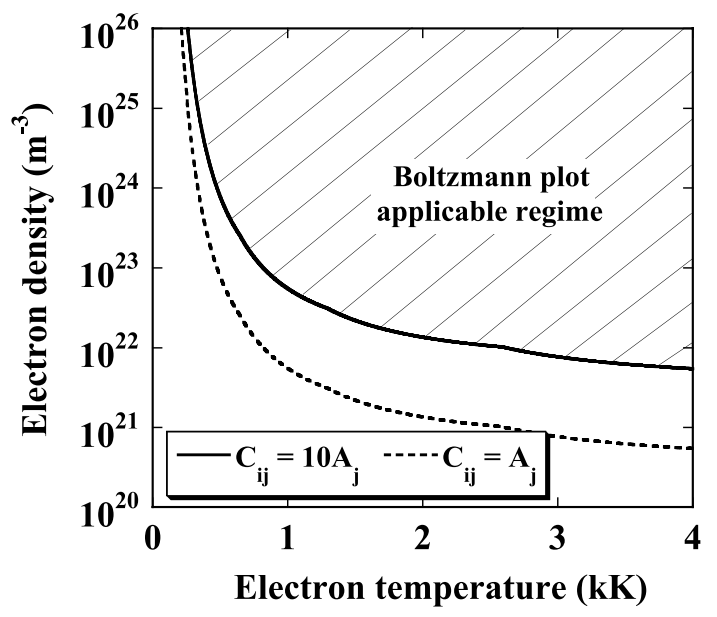

Fig. 3 Plasma parameter region (shaded area) defined by $C_{i j}>$ $10 A_{j}$, in which the Boltzmann plot method is applicable for $\operatorname{ArI} 555.87 \mathrm{~nm}$.

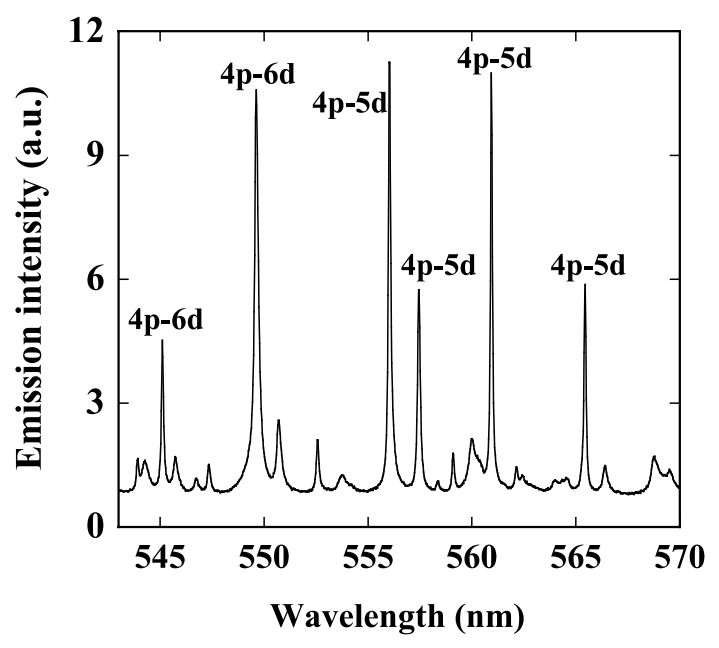

Fig. 4 ArI line spectrum radiated from Ar plasma at a gas pressure of $90 \mathrm{kPa}$.

Table 2 Atomic data of Ar [17].

\begin{tabular}{ccccccc}
\hline \hline$\lambda(\mathrm{mm})$ & $A_{k i}\left(10^{8} \mathrm{~s}^{-1}\right)$ & Error $(\%)$ & $E_{i}(\mathrm{eV})$ & $E_{k}(\mathrm{eV})$ & $g_{i}-g_{k}$ & Transition \\
\hline 545.16 & $4.70 \times 10^{-3}$ & 50 & 12.907 & 15.180 & $5-7$ & $4 \mathrm{p}-7 \mathrm{~s}$ \\
549.58 & $1.69 \times 10^{-2}$ & 25 & 13.076 & 15.331 & $7-9$ & $4 \mathrm{p}-6 \mathrm{~d}$ \\
555.87 & $1.42 \times 10^{-2}$ & 25 & 13.094 & 14.955 & $3-5$ & $4 \mathrm{p}-6 \mathrm{~d}$ \\
557.25 & $6.60 \times 10^{-3}$ & 25 & 11.623 & 13.480 & $5-7$ & $4 \mathrm{~s}-6 \mathrm{~d}$ \\
560.67 & $2.20 \times 10^{-2}$ & 25 & 12.907 & 14.742 & $3-3$ & $4 \mathrm{p}-6 \mathrm{~d}$ \\
565.07 & $3.20 \times 10^{-2}$ & 25 & 13.171 & 15.003 & $3-1$ & $4 \mathrm{p}-6 \mathrm{~d}$ \\
\hline \hline
\end{tabular}

method in a wavelength range of $547-570 \mathrm{~nm}$. The atomic data of argon line spectrum in these wavelength ranges are displayed in Table 2 [29].

Figure 4 shows the light emission spectrum of $\mathrm{Ar}$ plasma at a gas pressure of $90 \mathrm{kPa}$ and an absorbed power of about $8.4 \mathrm{~kW}$ in the wavelength range of $547-570 \mathrm{~nm}$. Figure 5 shows the Boltzmann plot of the ArI line spectrum for $545.16,549.58,555.87,557.25,560.67$, and $565.07 \mathrm{~nm}$

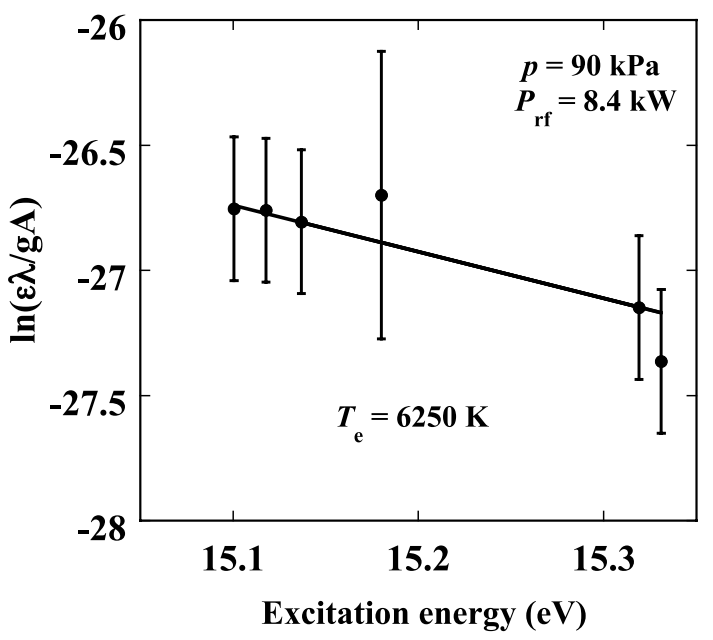

Fig. 5 Boltzmann plot of the ArI line spectrum for 545.16, 549.58, 555.87, 557.25, 560.67, and $565.07 \mathrm{~nm}$ with $90 \mathrm{kPa}, 8.4 \mathrm{~kW}$.

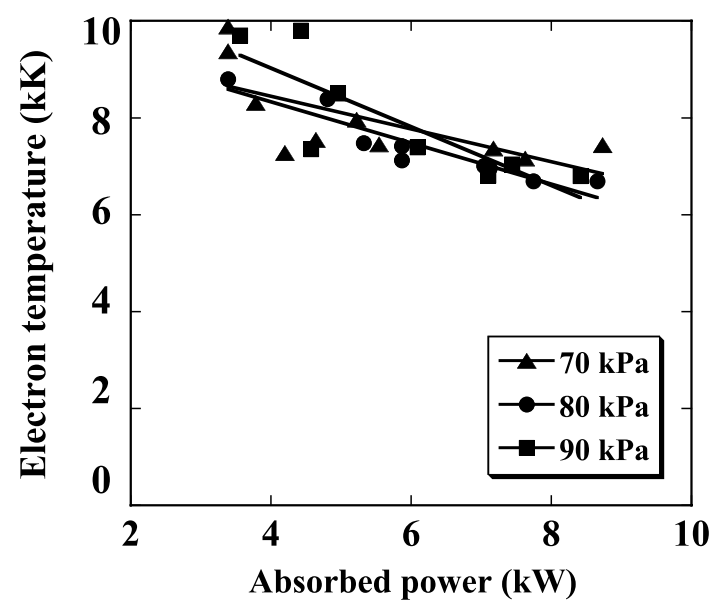

Fig. 6 Absorbed power dependence of electron temperature calculated in the wavelength range for use of the Boltzmann plot method at gas pressures of 70,80 , and $90 \mathrm{kPa}$.

at $90 \mathrm{kPa}$ and $8.4 \mathrm{~kW}$. The error bar shown in Fig. 5 represents the measurement error of the $A_{k i}$ coefficient of the ArI lines. The electron temperature measured from the slope of the straight line of Fig. 5 are found to be about 6,250 K at a gas pressure of $90 \mathrm{kPa}$. A similar calculation was performed for different power absorption cases at gas pressures of 70,80, and $90 \mathrm{kPa}$ and is plotted in Fig. 6, where it can be observed that the electron temperature decreases slightly with an increase in the rf power absorbed by the plasma. However, this phenomenon is generally considered unlikely. Therefore, in the present rf-inductive plasmas, the Boltzmann plot method cannot provide an exact measurement of electron temperature, because in this temperature range the electron density may be relatively low, not satisfying the requirements of the calculation criteria $\left(C_{i j} \gg A_{j}\right)$. Therefore, in this study, an alternative method using a combination of the Stark broadening measurement 
and the Saha-Boltzmann equation has been demonstrated to determine the electron temperature of rf-inductive $\mathrm{Ar}$ thermal plasmas, which will be described in Sec. 5 .

\section{Electron Density Measurement Us- ing Stark Width}

Collisions between radiating atoms and surrounding particles can lead to a broadening of the lines. Such collisional broadening can be classified into three groups: (a) Stark broadening, (b) van der Waals broadening, and (c) resonance broadening. Stark broadening is the statistical shift of the spectral lines by micro-fields produced by electrons and ions surrounding the radiating atoms, which is utilized for plasma density measurement [30-32]. In this section, the electron density is evaluated using Stark broadening of the ArI (549.58 nm) line.

On the other hand, van der Waals broadening occurs from the dipole interaction between radiating atoms and the induced dipole of the surrounding ground state atoms, which will dominate in high pressure discharges. From Ref. [33], van der Waals broadening for the ArI $(549.58 \mathrm{~nm})$ line can be estimated to within $0.01 \mathrm{~nm}$ at a gas temperature of $7,000 \mathrm{~K}$, which is one order of magnitude smaller than the observed line width. Resonance broadening occurs between neutrals of the same species and is confined to transitions with the upper and lower states connected by electric dipole transition (resonance line) to the ground state. Thus, resonance broadening is not expected to play a role for the line broadening of the $\operatorname{ArI}(549.58 \mathrm{~nm})$ line in our experiments.

To perform electron density measurement in rfinductive Ar plasmas from Stark broadening, the following expressions are used [34]:

$$
\begin{aligned}
d & =2[1+1.75 \alpha(1-0.75 r)] \omega \\
\alpha & =\left(\frac{N_{\mathrm{e}}}{N_{\mathrm{e}}^{0}}\right)^{1 / 4} \alpha_{n}(T), \\
\omega & =\frac{N_{\mathrm{e}}}{N_{\mathrm{e}}^{0}} \omega_{n}(T), \\
r & =\frac{\rho_{r}}{\rho}=\frac{(4 \pi N / 3)^{1 / 3}}{\varepsilon_{0} k T /\left(N e^{2}\right)}=6^{1 / 3} \pi^{1 / 6}\left(\frac{e^{2}}{4 \pi \varepsilon_{0} k T}\right)^{1 / 2} N^{1 / 6}, \\
& =9 \times 10^{-3} \frac{N_{\mathrm{e}}^{1 / 6}}{\sqrt{T}}, \\
N_{\mathrm{e}}^{0} & =10^{22} \mathrm{~m}^{-3},
\end{aligned}
$$

where $\omega$ represents the spectrum broadening due to electron collision, $\alpha$ is the broadening due to the ion presence in the electric field, and $r$ is the mean distance between ions divided by the Debye length. Parameters $\omega_{n}(T)$ and $\alpha_{n}(T)$ were tabulated in Ref. [34] for different temperatures. The value of $\omega$ and $\alpha$ for the ArI $(549.58 \mathrm{~nm})$ line as a function of electron temperature is plotted in Fig. 7.

However, an exact measurement of Stark broadening is not possible unless the broadenings of the spectral line due to other effects such as the Doppler effect are consid-

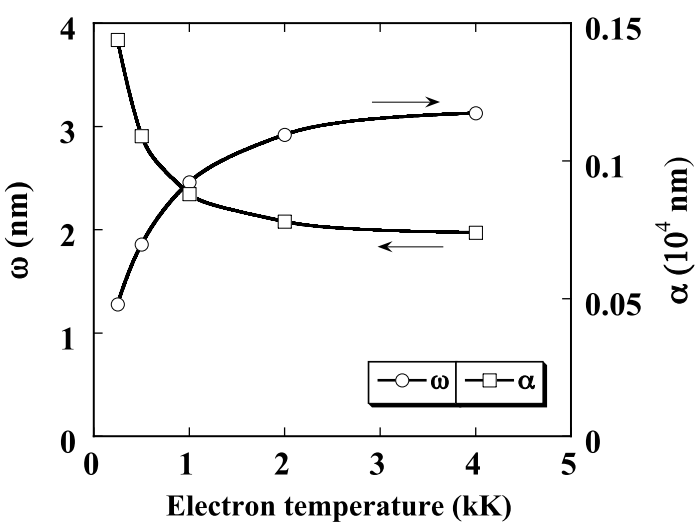

Fig. 7 Electron temperature dependence $\omega$ and $\alpha$ for the ArI $(549.58 \mathrm{~nm})$ line.

ered. Generally, apart from the Stark width, the instrumental width, natural width, and Doppler width are the main broadening mechanisms for the spectral lines of rfinductive Ar plasmas, which should be considered in order to measure the exact Stark width.

The instrumental width of a spectral line, arising from the resolution of a spectrometer, depends on the instrumental slit function. This instrumental width is generally expressed by the Gauss function [30-33]. In the present study, the instrumental width in a wavelength range of $300-600 \mathrm{~nm}$ is found to be $0.05-0.06 \mathrm{~nm}$, which cannot be neglected in the present case, and must be counted to determine the Stark broadening.

The natural broadening of a spectral line is the width arising from the spontaneous (natural) emission. This natural line shift, arising from uncertainty in the energy of the states involved in the transition, occurs when an excited atom is de-excited to a lower level by the spontaneous emission. The FWHM (Full Width at Half Maximum) natural line width, which is determined by the transition probability $A$, can be written in terms of wavelength $\lambda$ as [33]

$$
\Delta \lambda=\frac{\lambda^{2}}{2 \pi c} A .
$$

A typical natural line width corresponding to a transition probability of $10^{8} \mathrm{~s}^{-1}$ and a wavelength $549.58 \mathrm{~nm}$ calculated using Eq. (16) is found to be about $1.6 \times 10^{-5} \mathrm{~nm}$, which is very small compared with the instrumental width $(0.05-0.06 \mathrm{~nm})$, and can therefore be neglected in the present study.

Finally, Doppler broadening is the broadening of spectral lines due to the Dopller effect in which the thermal movement of atoms or molecules shifts the apparent frequency or wavelength of each emitter. The many different velocities of the emitting gas results in many small shifts, the cumulative effect of which is to broaden the line. The broadening is dependent only on the line's wavelength, the mass of the emitting particle, and the temperature, and can therefore be a very useful method for measuring the temperature of an emitting gas. The Doppler broadening in 
terms of wavelength can be written as [33]

$$
\Delta \lambda_{\text {Doppler }}=7.156 \times 10^{-7} \lambda_{0} \times\left(\frac{T}{W_{\mathrm{A}}}\right)^{1 / 2}[\mathrm{~m}] .
$$

The Doppler width corresponding to Ar plasma with an electron temperature of $10,000 \mathrm{~K}$ for the ArI $(549.58 \mathrm{~nm})$ line calculated using Eq. (17) is found to be $0.0062 \mathrm{~nm}$, which cannot be neglected in comparison with the instrumental width $(0.05-0.06 \mathrm{~nm})$, and therefore it is taken account of in determining the Stark broadening in the present study.

Now, it is noticed that the instrumental width and the Doppler width can be expressed by the Gauss function, while the Stark width is expressed by the Lorentz function [30-33]. Therefore, the profile of the spectral lines can be expressed by the Voigt function, which is the superposition of the Gauss function and the Lorentz function and can be expressed by [33]

$$
\begin{aligned}
f(\lambda)= & \frac{\Delta \lambda_{\text {Lorentz }}}{\pi^{3 / 2} \Delta \lambda_{\text {Gauss }}} \int_{0}^{\infty} \frac{1}{\left(x-\lambda_{\text {Line }}\right)^{2}+\Delta \lambda_{\text {Lorentz }}^{2}} \\
& \times \exp \left[-\left(\frac{x-\lambda_{\text {Line }}}{\Delta \lambda_{\text {Gauss }}}\right)^{2}\right] d x,
\end{aligned}
$$

where $\Delta \lambda_{\text {Lorentz }}, \Delta \lambda_{\text {Gauss }}$, and $\lambda_{\text {Line }}$ are the Lorentzian width, Gaussian width, and wavelength of the spectral line, respectively. Therefore, in order to evaluate the Stark width, it is necessary to remove the Gaussian element from the Voigt function. Since both the Doppler width and the instrumental width are gauss profiles, these two widths can be expressed by a single width by overlapping them with each other, which can be expressed by [33]

$$
\Delta \lambda_{\text {Gauss }}=\sqrt{\Delta \lambda_{\text {Instrument }}^{2}+\Delta \lambda_{\text {Doppler }}^{2}}
$$

where $\Delta \lambda_{\text {Instrument }}$ and $\Delta \lambda_{\text {Doppler }}$ are the instrumental width and Doppler width, respectively. In this study, the instrumental width and the Doppler width are found to be about $0.05 \mathrm{~nm}$ and $0.0062 \mathrm{~nm}$, respectively. Therefore, the Gaussian width calculated using Eq. (19) is found to be approximately $0.05 \mathrm{~nm}$.

In the present experiment, ArI $549.58 \mathrm{~nm}$ is chosen to estimate the Stark width because it is a strong and wellisolated line in the neutral argon spectrum, as shown in Fig. 4. However, it is necessary to perform deconvolution in order to evaluate the Stark width because the FWHM of the ArI line is a result of the convolution of the Lorentz profile (the Stark effect) and the Gaussian profile (the Doppler effect and instrumental broadening). A superimposed Gaussian width of $0.05 \mathrm{~nm}$ is used to perform the deconvolution, which is plotted in Fig. 8 (a). Finally, the Stark width is estimated by fitting the Lorentz function after performing the deconvolution. The Lorentzian width and Gaussian width are substituted in Eq. (18) in order to verify the deconvolution by comparing the ArI $(549.58 \mathrm{~nm})$ line spectrum with the Voigt function, as shown in Fig. 8 (b),
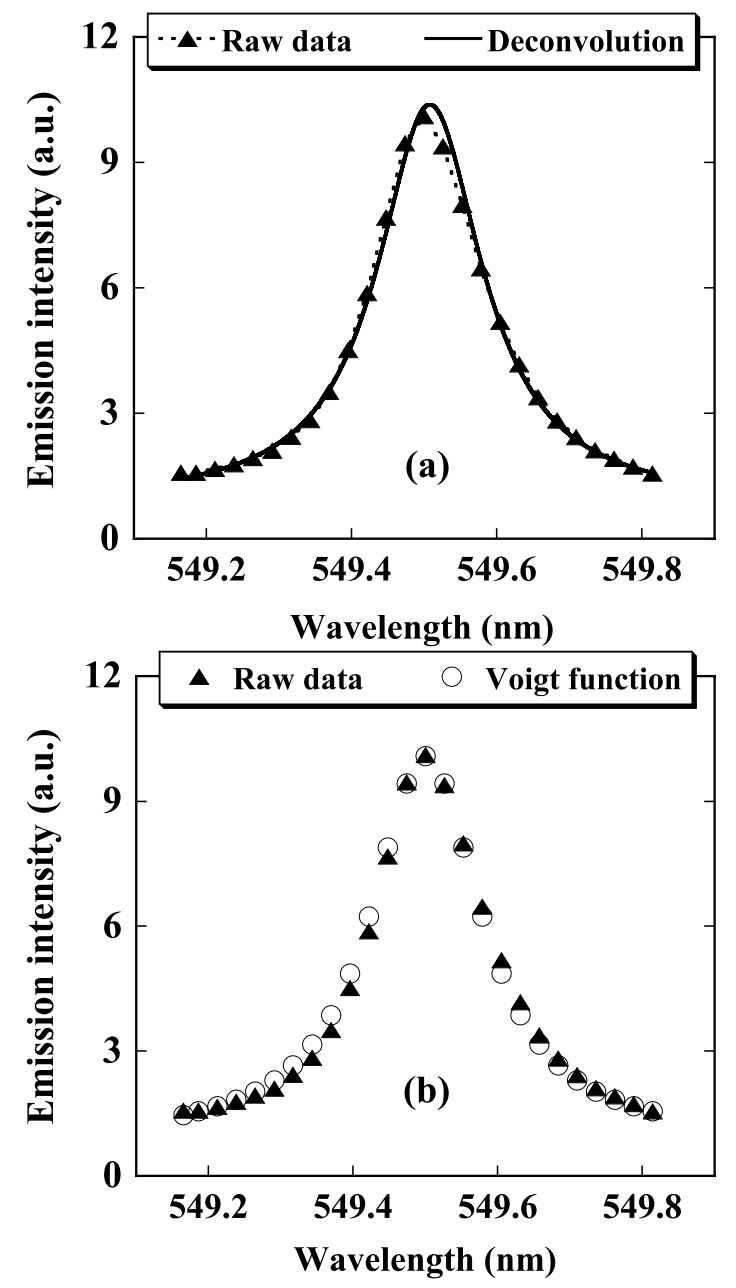

Fig. 8 Deconvolution of ArI (549.58 nm) line (a) and comparison of spectrum data and Voigt function (b).

where the Lorentzian width is chosen to be $0.17 \mathrm{~nm}$ along with the superimposed Gaussian width of $0.05 \mathrm{~nm}$. Figure 8 (b) demonstrates that the raw spectrum profile and the Voigt function are in good agreement, which justifies the evaluation of the Lorentzian width by deconvolution.

Using the process discussed in the above paragraphs, the absorbed power dependence of the Stark widths of Ar plasma for the ArI $(549.58 \mathrm{~nm})$ line at a gas pressure of 70,80 , and $90 \mathrm{kPa}$ have been estimated and are plotted in Fig. 9. It is observed that the Stark width increases with an increase of absorbed rf power at a power range of 4$9 \mathrm{~kW}$ in which the value of Stark widths lie between 0.1 and $0.2 \mathrm{~nm}$. In the present study, the plasma parameters of rf-inductive Ar thermal plasmas are calculated using an average Stark width value of $0.16 \mathrm{~nm}$, and are described in the following section.

The relation between electron temperature and electron density, determined by the expressions written in Eqs. (11)-(15), at a Stark width of $0.16 \mathrm{~nm}$ for the ArI $(549.58 \mathrm{~nm})$ line, is plotted in Fig. 10. From this figure, the electron densities of rf-inductive Ar plasmas at a gas 


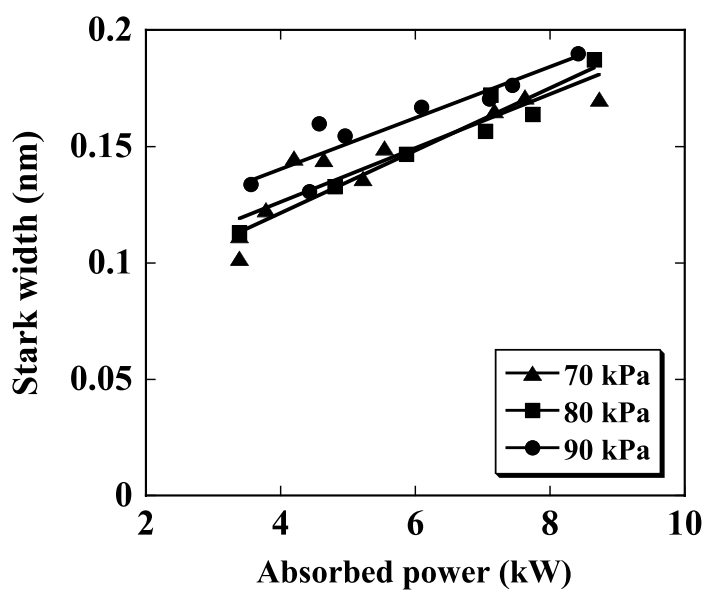

Fig. 9 Absorbed power dependence of Stark width of Ar plasma for the ArI $(549.58 \mathrm{~nm})$ line at gas pressures of 70, 80, and $90 \mathrm{kPa}$.

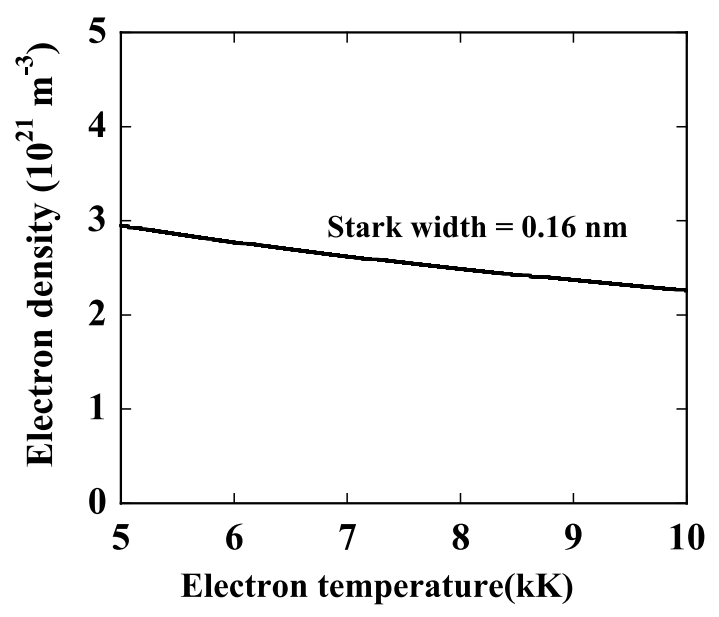

Fig. 10 Electron temperature dependence of electron density when the Stark width of the ArI $(549.58 \mathrm{~nm})$ line is $0.16 \mathrm{~nm}$.

pressure of $90 \mathrm{kPa}$ in an electron temperature range of $5,000-10,000 \mathrm{~K}$ are found to be $2.25 \times 10^{21}-3 \times 10^{21} \mathrm{~m}^{-3}$. This electron density range is much smaller than the range $\left(10^{23}-10^{24}\right)$ in which the Boltzmann plot is applicable (see Fig. 3). Therefore, the Boltzmann plot method of determining the exact electron temperature is not applicable in the present case.

\section{Plasma Parameter Measurement Using Stark Width and the Saha- Boltzmann Equation}

Since the Boltzmann plot method does not provide an exact electron temperature measurement of the generated plasmas under the present condition, the Saha-Boltzamann equation can be used to determine the electron temperature, which under a LTE condition can be expressed by [15]

$$
\frac{x^{2}}{1-x^{2}}=\frac{2 p m_{\mathrm{e}}}{h^{2}} \frac{(k T)^{5 / 2}}{p} \frac{g_{\mathrm{A}^{+}} \times g_{\mathrm{e}}}{g_{\mathrm{A}}} \mathrm{e}^{-e \varphi_{i} /(k T)},
$$

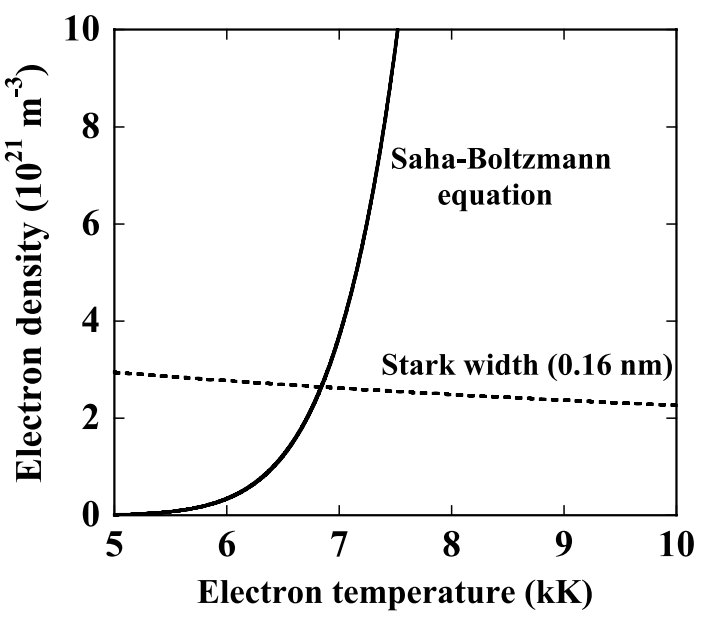

Fig. 11 Determination of plasma parameters using the SahaBoltzmann equation and Stark width of $0.16 \mathrm{~nm}$ for $\mathrm{rf}$ inductive Ar plasma at a gas pressure $80 \mathrm{kPa}$.

where $x$ is the degree of ionization, $p$ is the total gas pressure, $m_{\mathrm{e}}$ is the electron mass, $e$ is the electronic charge $\left(1.6 \times 10^{-19} \mathrm{~J}\right), \varphi_{i}$ is the ionization energy, $T$ is the electron temperature, and $g_{\mathrm{A}}, g_{\mathrm{A}^{+}}$, and $g_{\mathrm{e}}$ represent the statistical weights of the neutral particle, ion, and electron, respectively. However, plasmas must reach a LTE condition in order to apply the Saha-Boltzmann equation, and in order to have the plasma in a LTE state, the calculation criteria $C_{i j} \gg A_{j}$ must be satisfied. However, in our present rf-inductive plasmas, the condition $C_{i j} \gg A_{j}$ is not satisfied as described in the previous sections. Nevertheless, in the present study we want to determine an approximate value of the electron temperature, $T_{\mathrm{e}}$, of rf-inductive plasmas in the atmospheric pressure range, which gives at least better results than those achieved using the Boltzmann plot method. Therefore, an alternative method employing combination of the Saha-Boltzamann equation and Stark broadening measurement has been applied to determine the plasma parameters $\left(T_{\mathrm{e}}\right.$ and $\left.n_{\mathrm{e}}\right)$.

Figure 11 shows the plasma parameters of rf-inductive Ar plasmas at a gas pressure of $80 \mathrm{kPa}$ determined by the Saha-Boltzmann equation in combination with the Stark broadening measurement at a Stark width of $0.16 \mathrm{~nm}$ for the ArI $(549.58 \mathrm{~nm})$ line. The intersection of two graphs (Fig. 11) gives an electron density of $2.65 \times 10^{21} \mathrm{~m}^{-3}$ and an electron temperature of $6,850 \mathrm{~K}$. Similar calculations for the measurement of electron temperature and electron density have been performed for different power absorption cases and plotted in Fig. 12. From this figure, it is seen that the electron temperature increases from 7,000 to $7,500 \mathrm{~K}$ while the electron density increases from $3 \times 10^{21}$ to $4 \times 10^{21} \mathrm{~m}^{-3}$ when the effective absorbed $\mathrm{rf}$ power doubles from 4 to $8 \mathrm{~kW}$ at gas pressures of 70,80 , and $90 \mathrm{kPa}$. Therefore, the new method (the use of the SahaBoltzmann equation in combination with Stark broadening) provides more accurate results than those achieved by 


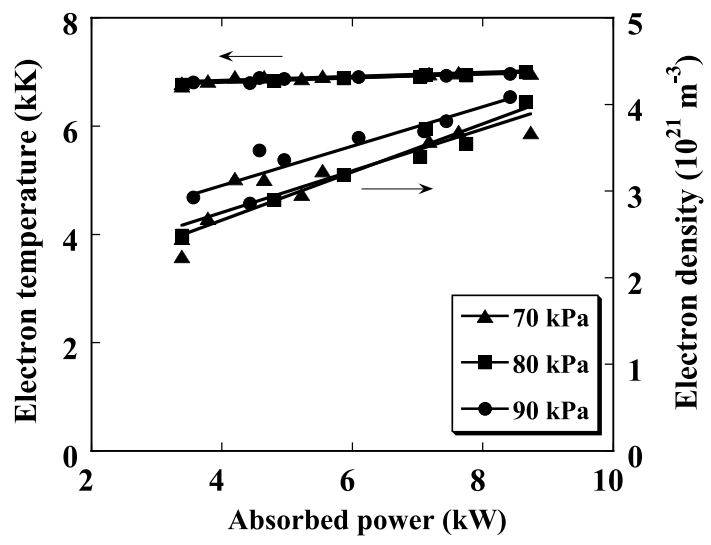

Fig. 12 Absorbed power dependence of electron temperature and electron density using Stark broadening measurement and the Saha-Boltzmann equation at gas pressures of 70 , 80 , and $90 \mathrm{kPa}$.

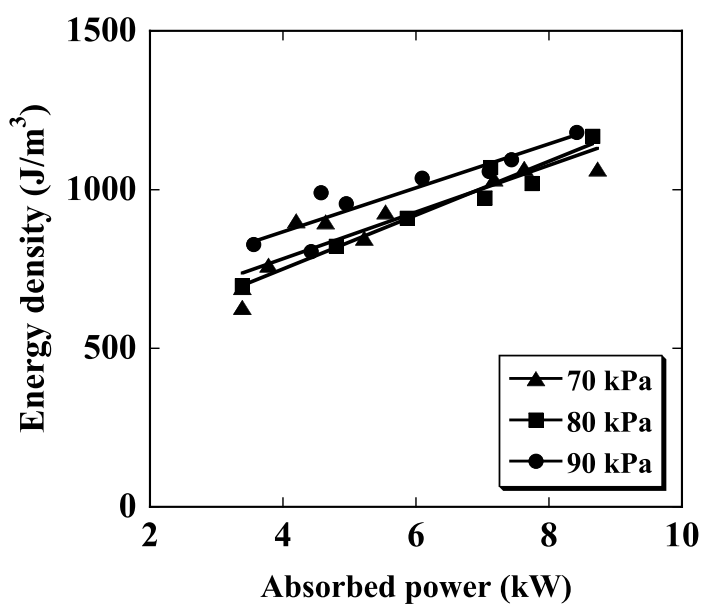

Fig. 13 Absorbed power dependence of plasma energy density.

the Boltzmann plot method, since by using this method both the electron temperature and electron density are increased with an increase of absorbed power as expected. This can be understood clearly if we consider the plasma energy density, which is written by

$$
\varepsilon=3 n_{\mathrm{e}} k T_{\mathrm{e}},
$$

where $n_{\mathrm{e}}$ is the electron density, $T_{\mathrm{e}}$ is the electron temperature, and $k$ is the Boltzmann constant. The effective absorbed rf power dependence of the plasma energy density is plotted in Fig. 13, from where it is seen that the plasma energy density increases from 800 to $1,200 \mathrm{~J} / \mathrm{m}^{3}$ with an increase of the effective absorbed rf power from 4 to $8 \mathrm{~kW}$ at gas pressures of 70,80 , and $90 \mathrm{kPa}$.

\section{Summary}

The validity of electron temperature measurement using the Boltzmann plot method has been investigated in a radio frequency (rf) inductive discharge in the atmospheric pressure range. The parameter region of electron temperature and electron density, in which the Boltzmann plot method is applicable, has been analyzed by a comparison of the spontaneous emission and collisional excitation to the excited states. The analyses reveal that in a radio frequency inductive discharge even in the atmospheric pressure range with a high electron-neutral collision frequency, it is necessary to verify calculation criteria $\left(C_{i j} \gg A_{j}\right)$ along with the LTE condition in order to apply the Boltzmann plot method for evaluating the electron temperature, $T_{\mathrm{e}}$.

The electron temperature measured using the Boltzmann plot method at a gas pressure of $90 \mathrm{kPa}$ is found to be $7,600 \mathrm{~K}$. On the other hand, the electron density measured using the Stark width $(0.16 \mathrm{~nm}$ in the present case) is found to be $2.65 \times 10^{21} \mathrm{~m}^{-3}$, which are much smaller than the $10^{23}-10^{24} \mathrm{~m}^{-3}$ where the Boltzmann plot is applicable (see Fig. 3). Therefore, in our rf-inductive plasma near the atmospheric pressure range, the Boltzmann plot method does not provide an exact electron temperature measurement because of relatively low electron density, which does not satisfy the measurement criteria $\left(C_{i j} \gg A_{j}\right)$. An alternative method employing a combination of Stark broadening measurement and the Saha-Boltzmann equation has been demonstrated to determine plasma parameters $\left(T_{\mathrm{e}}\right.$ and $\left.n_{\mathrm{e}}\right)$ of rf-inductive thermal plasmas, and it was found that this method provides more accurate results than those achieved through the use of the Boltzmann plot method alone. Using this method, it is observed that the electron temperature increases from about 7,000 to $7,500 \mathrm{~K}$ while the electron density increases from $3 \times 10^{21}$ to $4 \times 10^{21} \mathrm{~m}^{-3}$ when the effective absorbed rf power doubles from 4 to $8 \mathrm{~kW}$ at gas pressures of 70, 80, and $90 \mathrm{kPa}$.

[1] M. Amakawa, K. Adachi and S. Yasui, Trans. IEE, Japan 119-A, 357 (1999). [in Japanese]

[2] R. Benocci, G. Bonizzoni and E. Sindoni ed., Proc. Intl. School Plasma Phys. (World Scientific Publishing Singapore, 1996).

[3] M. Sakano and M. Tanaka, J. Plasma Fusion Res. 76, 748 (2000). [in Japanese]

[4] J.V.R. Heberlein, W.G. Melilli, S.V. Dighe and W.H. Reed, Proc. Workshop Industrial Applications, M. Boulos ed., Pugnochuso, Italy (1989) p.1.

[5] R.C. Eschenbach, Proc. Workshop Industrial Applications, M. Boulos ed., Pugnochuso, Italy (1989) p.127.

[6] S.L. Camacho, Proc. 1st Intl. EPRI Plasma Symp., EPRI Center for Materials Production (1990) Report No. CM909.

[7] E.L. Quiros, Brazilian J. Phys. 34, 1587 (2004).

[8] P.C. Kong and E. Pfender, Materials Processing-Theory and Practice (Elsevier, Amsterdam, 1992).

[9] J. Reece Roth, Industrial Plasma Engineering-Volume 2: Applications to Nonthermal Plasma Processing (Institute of Physics Publishing, Bristol and Philadelphia, 2001).

[10] M. I. Boulos, P. Fauchais and E. Phender, Thermal Plasmas: Fundamentals and Applications (Plenum Press, New York, 1994).

[11] J. Reece Roth, Industrial Plasma Engineering-Volume 1: Principles (Institute of Physics Publishing, Bristol, 1995).

[12] M.A. Razzak, S. Takamura, Y. Uesugi and N. Ohno, J. 
Plasma Fusion Res. 81, 204 (2005).

[13] M.A. Razzak, S. Takamura and Y. Uesugi, J. Appl. Phys. 96, 4771 (2004).

[14] M.A. Razzak, K. Kondo, Y. Uesugi, N. Ohno and S. Takamura, J. Appl. Phys. 95, 427 (2004).

[15] M.A. Razzak, S. Takamura and Y. Uesugi, IEEE Trans. Plasma Sci. 33, 284 (2005).

[16] Y. Uesugi, M.A. Razzak, K. Kondo, Y. Kikuchi, S. Takamura, T. Imai and M. Toyoda, J. Plasma Fusion Res. 79, 1062 (2003).

[17] Y. Uesugi, H. Ukai, M.A. Razzak and S. Takamura, IEEJ Trans. Fundamentals Materials 125, 749 (2005).

[18] J. Kim, K.S. Nam and K.C. Jungling, IEEE Trans. Electron Devices 44, 1022 (1997).

[19] M. Tadokoro, A. Itoh, N. Nakano, Z.L. Petrovic and T. Makabe, IEEE Trans. Plasma Sci. 26, 1724 (1998).

[20] N.A. Gatsonis, L.T. Byrne, J.C. Zwahlen, E.J. Pencil and H. Kamhawi, IEEE Trans. Plasma Sci. 32, 2118 (2004).

[21] T. Trottenberg, B. Brede, D. Block and A. Piel, IEEE Trans. Plasma Sci. 32, 742 (2004).

[22] Y. Tanaka and T. Sakuta, Plasma Sources Sci. Technol. 12, 69 (2003).

[23] K.C. Paul, T. Ishigaki, J. Mostaghimi and T. Sakuta, J. Appl. Phys. 93, 8867 (2003).
[24] Y. Tanaka and T. Sakuta, J. Physics D: Appl. Phys. 35, 2149 (2002).

[25] T. Sakuta, K.C. Paul, M. Katsuki and T. Ishigaki, J. Appl. Phys. 85, 1372 (1999).

[26] T. Sakuta, N. Sakashita, T. Yoshida, T. Takashima and M. Miyamoto, IEEE Trans. Plasma Sci. 25, 1029 (1997).

[27] H.R. Griem, Principles of Plasma Spectroscopy (Cambridge University Press, 1997).

[28] G.V. Marr, Plasma Spectroscopy (Elsevier Publishing Company, Amsterdam, 1968).

[29] http://www.physics.nist.gov/cgi-bin/AtData/ines_form (NIST Atomic Spectra Database Lines Form).

[30] Sobelman, L.A. Vainshtein and E.A. Yukov, Excitation of Atoms and Broadening of Spectral Lines (Springer-Verlag Berlin Heidelberg, New York, 1979).

[31] S. Pellerin, K. Musiol, B. Pokrzywka and J. Chapelle, J. Phys. B: Atomic, Molecular, Optical Phys. 29, 3911 (1996).

[32] H.R. Griem, Spectral Line Broadening by Plasma (Academic Press, New York, 1974).

[33] P.S. Moussounda and P. Ranson, J. Phys. B: At. Mol. Phys. 20, 949 (1987).

[34] H.R. Griem, Plasma Spectroscopy (McGraw Hill, New York, 1964). 